\title{
Partial Bi-Semimodules over Partial Semirings
}

\author{
V. Amarendra Babu ${ }^{1}$, M. Srinivasa Reddy ${ }^{2, *}$, P.V.Srinivasa Rao ${ }^{2}$ \\ ${ }^{1}$ Department of Mathematics, Acharya Nagarjunana University, Guntur, 522510, Andhra Pradesh, India \\ ${ }^{2}$ D. V. R \& Dr. H. S. MIC College of Technology, Kanchikacherla, 521180, Krishna(dt), Andhra Pradesh, India \\ *Corresponding Author: maths4444@gmail.com
}

Copyright (C 2014 Horizon Research Publishing All rights reserved.

\begin{abstract}
A partial semiring is a structure possessing an infinitary partial addition and a binary multiplication, subject to a set of axioms. The partial functions under disjoint-domain sums and functional composition is a partial semiring. In this paper we introduce the notions of ( $R, S)$ - partial bi-semimodule and ( $R, S)$ - homomorphism of $(R, S)$ - partial bi-semimodules and extended the results on partial semimodules over partial semirings by P. V. Srinivasa Rao [8] to ( $R, S)$ - partial bi-semimodules.
\end{abstract}

Keywords ( $R, S)$ - Partial Bi-Semimodule, $(N: M),(R, S)$ - Homomorphism, Bourne Relation, Steady ( $R, S)$ Homomorphism And Absorbing Subbi-Semimodule.

\section{Introduction}

Partially defined infinitary operations occur in the contexts ranging from integration theory to programming lanugage semantics. The general cardinal algebras studied by Tarski in 1949, $\sum$ - structures studied by Higgs in 1980, Housdorff topoligical commutative groups studied by Bourbaki in 1966, sum-ordered partial monoids and sum-ordered partial semirings studied by Arbib, Manes, Benson and Streenstrup are some of the algebraic structures of the above type.

The study of $\operatorname{pfn}(D, D)$ (the set of all partial functions of a set $D$ to itself), $M f n(D, D)$ (the set of all multi functions of a set $D$ to itself) and $\operatorname{Mset}(D, D)$ (the set of all total functions of a set $D$ to the set of all finite multi sets of $D$ ) play an important role in the theory of computer science, and to abstract these structures Manes and Benson[6] introduced the notion of sum ordered partial semirings(so-rings). Motivated by the work done in partially-additive semantics by Arbib, Manes[3] and in the development of matrix theory of so-rings by Martha E. Streenstrup[7], G. V. S. Acharyulu[1] in 1992 studied the conditions under which an arbitrary so-ring becomes a $p f n(D, D), M f n(D, D)$ and $M \operatorname{set}(D, D)$. Continuing this study, P. V. Srinivasa Rao[9] in 2011 developed the ideal theory for so-rings and partial semimodules over partial semirings.

In this paper we introduce the notions of $(R, S)$ - partial bi-semimodule, $(R, S)$ - homomorphism and absorbing subbi-semimodules and we generalise the results of semirings (Jonathan S. Golan [4] ) and results of partial semirings (Srinivasa Rao. P.V [9]) to the class of $(R, S)$ - partial bi-semimodules .

\section{Preliminaries}

In this section we collect important definitions, results and examples which were already proved for our use in the next sections.

2.1 Definition. [6] A partial monoid is a pair $(M, \Sigma)$ where $M$ is a non empty set and $\sum$ is a partial addition defined on some, but not necessarily all families $\left(x_{i}: i \in I\right)$ in $M$ subject to the following axioms:

Unary Sum Axiom: If $\left(x_{i}: i \in I\right)$ is a one element family in $M$ and $I=\{j\}$, then $\sum\left(x_{i}: i \in I\right)$ is defined and equals $x_{j}$.

Partition - Associativity Axiom: If $\left(x_{i}: i \in I\right)$ is a family in $M$ and If $\left(I_{j}: j \in J\right)$ is a partition of $I$, then $\left(x_{i}: i \in I\right)$ is summable if and only if $\left(x_{i}: i \in I_{j}\right)$ is summable for every $j$ in $J$ and $\left(\sum\left(x_{i}: i \in I_{j}\right): j \in J\right)$ is 
summable, and $\sum\left(x_{i}: i \in I\right)=\sum\left(\sum\left(x_{i}: i \in I_{j}\right): j \in J\right)$.

2.2 Definition. [6] The sum ordering $\leq$ on a partial monoid $(M, \Sigma)$ is the binary relation $\leq$ such that $x \leq y$ if and only if there exists a $h$ in $M$ such that $y=x+h$, for $x, y \in M$.

2.3 Definition. [6] A partial semiring is a quadruple $(R, \Sigma, ; 1)$, Where $(R, \Sigma)$ is a partial monoid with partial addition $\sum$, $(R,, 1)$ is a monoid with multiplicative operation '. ' and unit ' 1 ', and the additive and multiplicative structures obey the following distributive laws:

If $\sum\left(x_{i}: i \in I\right)$ is defined in $R$, then for all $y$ in $R, \sum\left(y \cdot x_{i}: i \in I\right)$ and $\sum\left(x_{i} \cdot y: i \in I\right)$ are defined and $y \cdot\left[\sum_{i} x_{i}\right]=\sum_{i}\left(y \cdot x_{i}\right),\left[\sum_{i} x_{i}\right] \cdot y=\sum_{i}\left(x_{i} \cdot y\right)$.

2.4 Definition. [6] A sum-ordered partial semiring (or so-ring, in short), is a partial semiring in which the sum ordering is a partial ordering.

2.5 Definition. [1] Let $R$ be a so-ring. A subset $N$ of $R$ is said to be an ideal of $R$ if the following are satisfied:

$\left(\mathrm{I}_{1}\right)$ if $\left(x_{i}: i \in I\right)$ is a summable family in $R$ and $x_{i} \in N$ for every $i \in I$ then $\sum_{i} x_{i} \in N$, ( $\left.\mathrm{I}_{2}\right)$ if $x \leq y$ and $y \in N$ then $x \in N$, and

$\left(\mathrm{I}_{3}\right)$ if $x \in N$ and $r \in R$ then $x r, r x \in N$.

2.6 Definition. [2] A subset $N$ of a so-ring $R$ is said to be a bi-ideal of $R$ if the following are satisfied:

$\left(\mathrm{B}_{1}\right)$ if $\left(x_{i}: i \in I\right)$ is a summable family in $R$ and $x_{i} \in N$ for every $i \in I$ then $\sum_{i} x_{i} \in N$,

$\left(\mathrm{B}_{2}\right)$ if $x \leq y$ and $y \in N$ then $x \in N$, and

$\left(\mathrm{B}_{3}\right)$ if $x, y \in N$ and $r \in R$ then $x r y \in N$.

2.7 Definition. [2]A subset $N$ of a so-ring $R$ is said to be a partial bi-ideal of $R$ if the following are satisfied:

i. $\quad$ if $\left(x_{i}: i \in I\right)$ is a summable family in $R$ and $x_{i} \in N$ for every $i \in I$ then $\sum_{i} x_{i} \in N$, and

ii. if $x, y \in N$ and $r \in R$ then $x r y \in N$.

2.8 Definition. [3]Let $M_{1}, M_{2}$ be $(R, S)$ - partial bi-semimodules. Then a mapping $\phi: M_{1} \rightarrow M_{2}$ is said to be an addative mapping if $\phi\left(\bar{\Sigma}_{i} m_{i}\right)=\bar{\Sigma} \phi\left(m_{i}\right)$ for any summable family $\left(m_{i}: i \in I\right)$ in $M_{1}$.

2.9 Definition. [7] Let $(R, \Sigma, ; 1)$ be a partial semiring and $(M, \bar{\Sigma})$ be a partial monoid. Then $M$ is said to be a left partial semimodule over $R$ if there exists a function $*: R \times M \rightarrow M:(r, x) \mapsto r * x$ which satisfies the following axioms for $x,\left(x_{i}: i \in I\right)$ in $M$ and $r_{1}, r_{2},\left(r_{j}: j \in J\right)$ in $R$

i. $\quad$ if $\sum_{i} x_{i}$ exists then $r *\left(\sum_{i} x_{i}\right)=\bar{\Sigma}\left(r * x_{i}\right)$,

ii. if $\sum_{j} r_{j}$ exists then $\left(\sum_{j} r_{j}\right) * x=\bar{\Sigma}\left(r_{j} * x\right)$,

iii. $\quad r_{1} *\left(r_{2} * x\right)=\left(r_{1} \cdot r_{2}\right) * x$, and

iv. $\quad 1_{R} * x=x$.Anlogously, one can define right partial semimodules over $R$. Throughout this paper $R, S$ donote partial semirings.

\section{3. ( $R, S)$ - Partial Bi-Semimodules}

In this section we prove that $(0: M)_{R}=(0: m)_{R}$ for every nonzero $m$ in $M$ where $M$ is a bi-austere $(R, S)$ - partial bi-semimodule.

3.1. Definition. Let $R, S$ be partial semirings and $(M, \bar{\Sigma})$ be a partial monoid. Then $M$ is said to be an $(R, S)$ - partial bi-semimodule if it satisfies the following axioms:

i. $\quad M$ is a left partial semimodule over $R$,

ii. $\quad M$ is a right partial semimodule over $S$, and (iii) for any $r \in R, x \in M, s \in S, r * x * s \in M$.

3.2. Definition. Let $(M, \bar{\Sigma})$ be an $(R, S)$ - partial bi-semimodule. Then a non empty subset $N$ of $M$ is said to be a 
subbi-semimodule of $M$ if $N$ is closed under $\bar{\Sigma}$ and $*$.

3.3. Definition. Let $N$ be a subbi-semimodule of an $(R, R)$ - partial bi-semimodule $M$. Then define $(N: M)$ as $(N: M)=\{r \in R \mid r M R \subseteq N\}$.

3.4. Definition. Let $N$ be a subbi-semimodule of an $(R, S)$ - partial bi-semimodule $M$. Then define $(N: M)_{R}$ and $(N: M)_{S}$ as $(N: M)_{R}=\{r \in R \mid r M S \subseteq N\}$ and $(N: M)_{S}=\{s \in S \mid R M s \subseteq N\}$.

3.5. Remark. Let $N$ be a subbi-semimodule of an $(R, S)$ - partial bi-semimodule $M$. Then $(N: M)_{R}$ and $(N: M)_{S}$ are partial bi-ideals of $R$ and $S$ respectively.

Proof: Note that $(N: M)_{R}=\{r \in R \mid r M S \subseteq N\}$. First we prove that $(N: M)_{R}$ is a partial bi-ideal of $R$. Let $\left(x_{i}: i \in I\right)$ be a summable family in $R$ and each $x_{i} \in(N: M)_{R} \forall i \in I$. Then $\sum_{i} x_{i}$ exists and $x_{i} M S \subseteq N$ $\forall i \in I . \Rightarrow \sum_{i} x_{i} M S \subseteq N . \Rightarrow\left(\sum_{i} x_{i}\right) M S \subseteq N$ and hence $\sum_{i} x_{i} \in(N: M)_{R}$. Let $x, y \in(N: M)_{R}$ and $r \in R$. Then $x M S \subseteq N$ and $y M S \subseteq N$. Now $(x r y) M S=x r(y M S) \subseteq x r N \subseteq N . \Rightarrow x r y \in(N: M)_{R}$. Hence $(N: M)_{R}$ is a partial bi-ideal of $R$. Similarly we can prove that $(N: M)_{S}$ is a partial bi-ideal of $S$.

3.6. Remark. Let $N$ be a subbi-semimodule of an $(R, R)$-partial bi-semimodule $M$. Then $(N: M)$ is a partial bi-ideal of $R$.

3.7. Theorem. If $N$ and $N^{\prime}$ are subbi-semimodules of an $(R, S)$ - partial bi-semimodule $M$ and if $A, B$ are non empty subsets of $M$ then ( i ) $A \subseteq B \Rightarrow(N: B)_{R} \subseteq(N: A)_{R}$, (ii ) $\left(N \cap N^{\prime}: A\right)_{R}=(N: A)_{R} \cap\left(N^{\prime}: A\right)_{R}$, and ( iii ) if $\bar{\Sigma}(a, b)$ exists for all $a \in A, b \in B$ then $(N: A)_{R} \cap(N: B)_{R} \subseteq(N: A+B)_{R}$ with equality holding if $0_{M} \in A \cap B$.

Proof: (i). Suppose $A \subseteq B$ and let $x \in(N: B)_{R}$. Then $x B S \subseteq N . \Rightarrow x b S \subseteq N \quad \forall b \in B . \Rightarrow x a S \subseteq N$ $\forall a \in A . \Rightarrow x A S \subseteq N . \Rightarrow x \in(N: A)_{R}$. Hence $(N: B)_{R} \subseteq(N: A)_{R}$.

(ii). Note that $x \in\left(N \cap N^{\prime}: A\right)_{R} \Leftrightarrow x A S \subseteq N \cap N^{\prime} \Leftrightarrow x A S \subseteq N$ and $x A S \subseteq N^{\prime} \Leftrightarrow x \in(N: A)_{R}$ and $x \in\left(N^{\prime}: A\right)_{R} \Leftrightarrow x \in(N: A)_{R} \cap\left(N^{\prime}: A\right)_{R}$.

(iii). Suppose $\bar{\Sigma}(a, b)$ exists for all $a \in A, b \in B$. Then $A+B=\{\bar{\Sigma}(a, b) \mid a \in A, b \in B\}$ exists and nonempty. Let $x \in(N: A)_{R} \cap(N: B)_{R}$. Then $x A S \subseteq N$ and $x B S \subseteq N . \Rightarrow x(A+B) S \subseteq N . \Rightarrow x \in(N: A+B)_{R}$. $\Rightarrow(N: A)_{R} \cap(N: B)_{R} \subseteq(N: A+B)_{R} \quad$. Suppose $\quad 0_{M} \in A \cap B \quad$ and let $\quad x \in(N: A+B)_{R}$ $\Rightarrow x(A+B) S \subseteq N . \Rightarrow x A S+x B S \subseteq N$. Since $0_{M} \in A$ and $0_{M} \in B, 0+x B S \subseteq N$ and $x A S+0 \subseteq N$. $\Rightarrow x A S \subseteq N \quad$ and $\quad x B S \subseteq N \quad \Rightarrow x \in(N: A)_{R} \quad$ and $\quad x \in(N: B)_{R} \quad . \quad \Rightarrow x \in(N: A)_{R} \cap(N: B)_{R}$. $\Rightarrow(N: A+B)_{R} \subseteq(N: A)_{R} \cap(N: B)_{R}$. Hence $(N: A+B)_{R}=(N: A)_{R} \cap(N: B)_{R}$.

3.8. Definition. Let $A$ be a non empty subset of an $(R, S)$ - partial bi-semimodule $M$. Then the subbi-semimodule generated by $A$ is the intersection of all subbi-semimodules of $M$ containing $A$ and is denoted by $R A S$.

Here we are able to generalise the results of partial semimodules over the partial semirings.

3.9. Theorem. Let $M$ be an $(R, S)$-partial bi-semimodule. Then for any non empty subset $A$ of $M$, $R A S=\left\{\bar{\Sigma}_{i}\left(r_{i} * a_{i} * s_{i}\right) \mid r_{i} \in R, s_{i} \in S, a_{i} \in A, i \in I\right\}$

Proof: Take $\mathrm{T}=\left\{\bar{\Sigma}_{i}\left(r_{i} * a_{i} * s_{i}\right) / r_{i} \in R, s_{i} \in S, a_{i} \in A\right\}$. First we prove that $\mathrm{T}$ is a subbi-semimodule of $M$. Let $\left(x_{i}: i \in I\right)$ be a summable family in $M$ such that $x_{i} \in \mathrm{T}, i \in I$. Then $\bar{\Sigma} x_{i}$ exists and each $x_{i}=\bar{\Sigma}\left(r_{i_{j}} * a_{i_{j}} * s_{i_{j}}\right), r_{i_{j}} \in R, a_{i_{j}} \in A, s_{i_{j}} \in S . \Rightarrow \overline{\sum_{i}} x_{i}=\bar{\Sigma} \bar{\Sigma}_{i}\left(r_{i_{j}} * a_{i_{j}} * s_{i_{j}}\right)$ exists and is in T. Let $r \in R, s \in S$ and $\quad x \in \mathrm{T}$. Then $\quad r \in R, s \in S \quad$ and $\quad x=\bar{\Sigma}\left(r_{i} * a_{i} * s_{i}\right) \quad . \quad \Rightarrow r * x * s=r * \bar{\Sigma}\left(r_{i} * a_{i} * s_{i}\right) * s=$ $\sum_{i} r *\left(r_{i} * a_{i} * s_{i}\right) * s=\bar{\Sigma}\left(r \cdot r_{i}\right) * a_{i} *\left(s_{i} \cdot s\right) \in \mathrm{T}$. Hence $\mathrm{T}$ is a subbi-semimodule of $M$. We have 
$a=1_{R} * a * 1_{S} \forall a \in A$ and hence $A \subseteq T$. To prove that $\mathrm{T}$ is smallest, let $N$ be a subbi-semimodule of $M$ containing $A$ and let $x \in \mathrm{T}$. Then $x=\bar{\Sigma}\left(r_{i} * a_{i} * s_{i}\right), r_{i} \in R, a_{i} \in A, s_{i} \in S . \Rightarrow r_{i} \in R, a_{i} \in N, s_{i} \in S \forall i \in I$. $\Rightarrow x=\bar{\Sigma}\left(r_{i} * a_{i} * s_{i}\right) \in N$. Hence $\mathrm{T}$ is the smallest subbi-semimodule of $M$ containing $A$.

3.10. Remark. Let $M$ be an $(R, S)$ - partial bi-semimodule. Then the set $\sum_{i \in I} N_{i}$ of all possible sums of elements of $\bigcup_{i \in I} N_{i}$, is the smallest subbi-semimodule of $M$ containing each $N_{i}$.

3.11. Definition. A non empty subset $N$ of an $(R, S)$-partial bi-semimodule $M$ is said to be subtractive if and only if for any $m, m^{\prime} \in M, m+m^{\prime} \in N$ and $m \in N$ implies $m^{\prime} \in N$.

3.12. Definition. An $(R, S)$-partial bi-semimodule $M$ is said to be bi-austere if and only if $\{0\}$ and $M$ are the only subtractive subbi-semimodules of $M$.

3.13. Remark. Let $N$ be a subbi-semimodule of an $(R, S)$-partial bi-semimodule $M$ and $A$ be a non empty subset of $M$. Then $(N: A)_{R}=\bigcap\left\{(N: a)_{R} \mid a \in A\right\}$.

3.14. Theorem. If $M$ is a bi-austere $(R, S)$-partial bi-semimodule then $(0: M)_{R}=(0: m)_{R}$ for every non zero $m$ in $M$.

Proof: Since $(0: M)_{R}=\bigcap\left\{(0: m)_{R} \mid m \in M\right\}$, we have $(0: M)_{R} \subseteq(0: m)_{R}$ for every nonzero $m$ in $M$. Suppose $(0: m)_{R} \not \subset(0: M)_{R}$ for some nonzero $m$ in $M$. Then $(0: m)_{R} \not \subset\left(0: m^{\prime}\right)_{R}$ for some non zero $m^{\prime} \in M$. Take $N=\left\{x \in M \mid(0: m)_{R} \subseteq(0: x)_{R}\right\}$. Then $0 \neq m \in N$ and $0 \neq m^{\prime} \notin N$ and hence $\{0\} \subset N \subset M$. Now we prove that $\mathrm{N}$ is a subtractive subbi-semimodule of $M$. Let $\left(x_{i}: i \in I\right)$ be a summable family in $\quad M \quad$ and $\quad x_{i} \in N, i \in I \quad$. Then $\quad \bar{\Sigma}_{i} x_{i} \quad$ exists $\quad$ and $\quad(0: m)_{R} \subseteq\left(0: x_{i}\right)_{R} \forall i \in I$ $\Rightarrow(0: m)_{R} \subseteq \sum_{i}\left(0: x_{i}\right)=\left(0: \sum_{i} x_{i}\right)$ and hence $\bar{\Sigma}_{i} x_{i} \in N$. Let $r \in R, s \in S$ and $x \in N$. Then $r \in R, s \in S$ and $(0: m)_{R} \subseteq(0: x)_{R} . \Rightarrow(0: m)_{R} \subseteq(0: r * x * s)_{R}$ and hence $r * x * s \in N$. Hence $\mathrm{N}$ is a subbi-semimodule of $M$. To prove that $N$ is subtractive, let $x, y \in M$ such that $x \in N$ and $\bar{\Sigma}(x, y) \in N$. Then $(0: m)_{R} \subseteq(0: x)_{R}$ and $(0: m)_{R} \subseteq\left(0: \bar{\Sigma}(x, y)_{R}\right)$. Now let $r \in(0: m)_{R}$. Then $r \in(0: x)_{R}$ and $r \in(0: \bar{\Sigma}(x, y))_{R} . \Rightarrow r x S=0$ and $r(\bar{\Sigma}(x, y)) S=0 . \Rightarrow r x S=0$ and $\bar{\Sigma}(r x S, r y S)=0 . \Rightarrow \bar{\Sigma}(0, r y S)=0 . \Rightarrow r y S=0 . \Rightarrow r \in(0: y)_{R}$. $\Rightarrow(0: m)_{R} \subseteq(0: y)_{R}$ and hence $y \in N . \Rightarrow N$ is a non trivial subtractive subbi-semimodule of $M$, a contradiction. Hence $(0: M)_{R}=(0: m)_{R}$ for every non zero $m$ in $M$.

3.15. Remark. If $N$ is a subtractive subbi-semimodule of an $(R, S)$-partial bi-semimodule $M$ and $A$ is a non empty subset of $M$ then $(N: A)_{R}$ is a subtractive partial bi-ideal of $R$.

Proof: By the remark 3.5, $(N: A)_{R}$ is a partial bi-ideal of $R$. Let $x, y \in R \ni x+y \in(N: A)_{R}$ and $x \in(N: A)_{R}$. Then $x+y \in(N: a)_{R}$ and $x \in(N: a)_{R} \forall a \in A . \Rightarrow(x+y) a S \subseteq N$ and $x a S \subseteq N . \Rightarrow(x+y) a s \in N$ and $x a s \in N \quad \forall s \in S . \Rightarrow x a s+y a s \in N \quad$ and $\quad x a s \in N \quad \forall s \in S . \Rightarrow y a s \in N \quad \forall s \in S . \Rightarrow y A S \subseteq N$. $\Rightarrow y \in(N: A)_{R}$ and hence $(N: A)_{R}$ is a subtractive.

3.16. Definition. An $(R, S)$-partial bi-semimodule $M$ is said to be entire if and only if $r * m * s \neq 0_{M}$ whenever $0_{R} \neq r \in R, \quad 0_{S} \neq s \in S$ and $0_{M} \neq m \in M$.

3.17. Theorem. A partial semiring $R$ is entire if and only if there exists a non trivial entire $(R, R)$-partial bi-semimodule. Proof: If $R$ is entire then $R$ is a non trivial $(R, R)$-partial bi-semimodule. Suppose $\exists$ a non trivial entire $(R, R)$-partial bi-semimodule $\quad M$. Then $\exists 0_{M} \neq m \in M$. Let $\quad r, r^{\prime} \in R \ni r r^{\prime}=0 . \quad \Rightarrow\left(r r^{\prime}\right) * m *\left(r r^{\prime}\right)=0_{M}$. $\Rightarrow r *\left(r^{\prime} * m * r\right) * r^{\prime}=0_{M}$. Since $M$ is entire, $r=0$ or $r^{\prime}=0$. Hence $R$ is entire. 
3.18 Remark. Let $M$ be an $(R, S)$-partial bi-semimodule. If $N, N^{\prime}, N^{\prime \prime}$ are subbi-semimodules of $M$ such that $N$ is subtractive and $N^{\prime} \subseteq N$, then $N \cap\left(N^{\prime}+N^{\prime \prime}\right)=N^{\prime}+\left(N \cap N^{\prime \prime}\right)$.

Proof: Clearly $N \cap\left(N^{\prime}+N^{\prime \prime}\right) \supseteq N^{\prime}+\left(N \cap N^{\prime \prime}\right)$. Let $x \in N \cap\left(N^{\prime}+N^{\prime \prime}\right)$. Then $x \in N$ and $x \in N^{\prime}+N^{\prime \prime}$. $\Rightarrow x \in N, x=y+z$ for some $y \in N^{\prime}, z \in N^{\prime \prime} . \Rightarrow x=y+z \in N$ and $y \in N$. Since $N$ is subtractive, $z \in N$ $\Rightarrow x=y+z \in N^{\prime}+\left(N \cap N^{\prime \prime}\right)$. Hence the remark.

\section{4. $(R, S)$ - Homomorphisms and Absorbing Subbi-Semimodules}

In this section we introduce the notions of $(R, S)$ - homomorphism, Bourne relation, steady $(R, S)$-homomorphism and absorbing subbi-semimodules and study various characteristics of them.

4.1. Definition. Let $M$ be an $(R, S)$ - partial bi-semimodule and $\theta$ be an equivalence relation on $M$. Then $\theta$ is said to be an $(R, S)$ - congruence relation on $M$ if and only if it satisfies the following: ( i ) $\theta$ is closed under the additive operation of the product $(R, S)$ - partial bi-semimodule $M \times M$. i.e., if $\left(x_{i}: i \in I\right)$ and $\left(y_{i}: i \in I\right)$ are summable families in $M$ such that $\left(x_{i}, y_{i}\right) \in \theta$ then $\sum_{i \in I}\left(x_{i}, y_{i}\right) \in \theta$, ( ii $)$ if $r \in R, s \in S,(x, y) \in \theta$ then $(r * x * s, r * y * s) \in \theta$.

4.2. Definition. Let $(M, \bar{\Sigma}, *)$ be an $(R, S)$ - partial bi-semimodule and $\theta$ be an $(R, S)$ - congruence relation on $M$. Then their quotient is the structure $\left(M / \theta, \bar{\Sigma}^{\prime},\right)$ where $M / \theta=\left\{[x]_{\theta} / x \in M\right\} \quad\left([x]_{\theta}\right.$ is the equivalence class containing $x$ with respect to $\theta), \bar{\Sigma}^{\prime}$ and $\cdot$ are defined as follows:

A family $\left(\left[x_{i}\right]_{\theta}: i \in I\right)$ is summable in $M / \theta$ if and only if $\left(x_{i}: i \in I\right)$ is summable in $M$. Then we write $\bar{\Sigma}_{i}^{\prime}\left[x_{i}\right]_{\theta}=\left[\bar{\Sigma}_{i} x_{i}\right]_{\theta}$. And '. , is a function $R \times M / \theta \times S \rightarrow M / \theta:\left(r,[x]_{\theta}, s\right) \mapsto r \cdot[x]_{\theta} \cdot s$ where $r \cdot[x]_{\theta} \cdot s=[r * x * s]_{\theta} \quad \forall r \in R, s \in S$ and $x \in M$.

The following example shows that $M / \theta$ need not be an $(R, S)$ - partial bi-semimodule.

4.3. Example. We know that $(P(D), \Sigma$,$) is a partial semiring, where$ $\sum_{i} A_{i}=\left\{\begin{array}{ccc}\cup A_{i} & \text { if } & A_{i} \cap A_{j}=\phi \quad \forall i \neq j \\ \text { undefined, } & \text { otherwise. }\end{array}\right.$ and $A \cdot B=A \cap B$. Take $R:=S:=M:=P(D)$. Then $M$ is an $(R, S)$ - partial bi-semimodule.

Let $D=\{x, y\}$. Then $\theta=\{(\phi, \phi),(\{x\},\{x\}),(\{y\},\{y\}),(D, D),(\{x\}, D),(D,\{x\}),(\phi,\{y\}),(\{y\}, \phi)\}$ is an $(R, R)$ - congruence relation on $P(D)$. Now $P(D) / \theta=\{\bar{\phi}, \overline{\{x\}}\}$, where $\bar{\phi}=\{\phi,\{y\}\}=\overline{\{y\}}, \overline{\{x\}}=\{\{x\}, D\}=\bar{D}$. Here $\{x\}+\{y\}$ is defined. But $\{x, y\}+\{y\}$ is not defined and hence $\overline{\{x\}}+\overline{\{y\}}$ is not well defined. Hence $P(D) / \theta$ is not a partial bi-semimodule.

4.4. Remark. Let $\theta$ be an $(R, S)$ - congruence relation on an $(R, S)$ - partial bi-semimodule $M$. Then a necessary and sufficient condition for $M / \theta$ to be a partial semiring is that the family $\left(y_{i}: i \in I\right)$ is summable whenever $\left(x_{i}: i \in I\right)$ is summable and $x_{i} \theta y_{i}, i \in I$.

4.5. Definition. Let $N$ be a subbi-semimodule of an $(R, S)$ - partial bi-semimodule $M$. Then the Bourne relation $\equiv_{N}$ on $M$ is defined as $m \equiv_{N} m^{\prime} \Leftrightarrow$ there exists $n, n^{\prime} \in N$ such that $m+n=m^{\prime}+n^{\prime}$.

4.6. Definition. An $(R, S)$ - partial bi-semimodule $M$ is said to be a complete $(R, S)$-partial bi-semimodule if for any family $\left(m_{i}: i \in I\right)$ in $M, \bar{\Sigma}_{i} m_{i}$ is in $M$.

4.7. Remark. The Bourne relation $\equiv_{N}$ is an $(R, S)$ - congruence relation on a complete $(R, S)$-partial bi-semimodule $M$. 
Proof: Clearly $\equiv_{N}$ is closed under the additive operation of product $(R, S)$ - partial bi-semimodule $M$. Let $r \in R, s \in S$ and $\quad\left(m, m^{\prime}\right) \in \equiv_{N} . \quad \Rightarrow \exists n, n^{\prime} \in N \ni m+n=m^{\prime}+n^{\prime} \quad \Rightarrow r *(m+n) * s=r *\left(m^{\prime}+n^{\prime}\right) * s$ $\Rightarrow r * m * s+r * n * s=r * m^{\prime} * s+r * n^{\prime} * s$. Since $n, n^{\prime} \in N \quad$ and $N$ is a subbi-semimodule of $M$, $r * n * s, r * n^{\prime} * s \in N . \Rightarrow r * m * s \equiv_{N} r * m^{\prime} * s$. Hence $\equiv_{N}$ is an $(R, S)$ - congruence relation on $M$.

We denote the equivalence class of $m$ as $m / N$ and the quotient $M / \equiv_{N}$ by $M / N$.

4.8. Definition. Let $N$ be a subbi-semimodule of an $(R, S)$ - partial bi-semimodule $M$. Then the subtractive closure of $N$ is the intersection of all subtractive subbi-semimodules of $M$ containing $N$.

4.9. Remark. If $N$ is a subbi-semimodule of a complete $(R, S)$ - partial bi-semimodule $M$. Then $0 / N$ is the subtractive closure of $N$.

Proof: Note that $0 / N=\left\{m \in M \mid m \equiv_{N} 0\right\}=\{m \in M \mid \exists n \in N \ni m+n \in N\}$. First we prove that $0 / N$ is a subtractive subbi-semimodule of $M$. Let $\left(m_{i}: i \in I\right)$ be a summable family in $M$ such that $m_{i} \in 0 / N, i \in I$. Then $m_{i} \equiv_{N} 0 \forall i \in I . \Rightarrow \bar{\Sigma} m_{i} \equiv_{N} 0$ and hence $\bar{\Sigma}_{i} m_{i} \in 0 / N$. Let $r \in R, s \in S$ and $m \in 0 / N$. Then $r \in R, s \in S$ and $m \equiv_{N} 0_{M} . \Rightarrow r * m * s \equiv_{N} r * 0_{M} * s . \Rightarrow r * m * s \equiv_{N} 0_{M}$ and hence $r * m * s \in 0 / N$. Let $m, m^{\prime} \in M \ni m+m^{\prime} \in 0 / N \quad$ and $\quad m \in 0 / N \quad . \quad \Rightarrow\left(m+m^{\prime}\right) \equiv_{N} 0 \quad$ and $\quad m \equiv_{N} 0$ $\Rightarrow \exists n, n^{\prime} \in N \ni\left(m+m^{\prime}\right)+n \in N \quad$ and $\quad m+n^{\prime} \in N . \quad \Rightarrow \exists m+n+n^{\prime} \in N \ni m^{\prime}+\left(m+n+n^{\prime}\right) \in N$ $\Rightarrow m^{\prime} \equiv_{N} 0$ and hence $m^{\prime} \in 0 / N$. Therefore $0 / N$ is a subtractive subbi-semimodule of $M$. For any $a \in N, 0+a=a+0$ and hence $a \equiv_{N} 0$. Hence $a \in 0 / N . \Rightarrow N \subseteq 0 / N$. Now let $N^{\prime}$ be a subtractive subbi-semimodule of $M \ni N \subseteq N^{\prime}$. Let $m \in 0 / N$. Then $\exists n \in N \ni m+n \in N . \Rightarrow \exists n \in N^{\prime}$ э $m+n \in N^{\prime}$. Since $N^{\prime}$ is subtractive, $m \in N^{\prime}$ and hence $0 / N \subseteq N^{\prime}$. Hence the remark.

4.10. Remark. If $N$ is a subbi-semimodule of a complete $(R, S)$-partial bi-semimodule $M$. Then the congruence relations $\equiv_{N}$ and $\equiv_{0 / N}$ on $M$ coincide.

Proof: Since $N \subseteq 0 / N$. Clearly $\equiv_{N} \subseteq \equiv_{0 / N}$. Now let $m \equiv_{0 / N} m^{\prime}$. Then $\exists x, y \in 0 / N \ni m+x=m^{\prime}+y$. $\Rightarrow(m+x) / N=\left(m^{\prime}+y\right) / N . \Rightarrow m / N+x / N=m^{\prime} / N+y / N$. Since $x, y \in 0 / N . \Rightarrow x / N=0 / N$ and $y / N=0 / N . \Rightarrow m / N=m^{\prime} / N . \Rightarrow m \equiv_{N} m^{\prime}$ and hence $\equiv_{N} \subseteq \equiv_{0 / N}$.

4.11. Definition. Let $M_{1}, M_{2}$ be $(R, S)$ - partial bi-semimodules. Then a mapping $\phi: M_{1} \rightarrow M_{2}$ is said to be an $(R, S)$-mapping if $\phi(r * x * s)=r * \phi(x) * s \forall x \in M, r \in R, s \in S$.

4.12. Definition. A mapping $\phi: M_{1} \rightarrow M_{2}$ is called an $(R, S)$-homomorphism of $(R, S)$ - partial bi-semimodules $M_{1}$, $M_{2}$ if ( i ) $\phi$ is an additive mapping, and ( ii ) $\phi$ is an $(R, S)$ - mapping.

4.13. Definition. Let $\phi: M_{1} \rightarrow M_{2}$ be an $(R, S)$-homomorphism of $(R, S)$ - partial bi-semimodules. Then the kernel of $\phi$ is $\operatorname{ker} \phi=\left\{x \in M_{1} \mid \phi(x)=0\right\}$, for any subset $M$ of $M_{1}, \phi M=\{\phi(m) \mid m \in M\}$ and for any $y \in M_{2}, \phi^{-1}(y)=\left\{x \in M_{1} \mid \phi(x)=y\right\}$.

4.14. Theorem. Let $M$ be a complete $(R, S)$ - partial bi-semimodule. Then a subset $N$ of $M$ is subtractive subbi-semimodule if and only if there exists an $(R, S)$ - homomorphism $\alpha: M \rightarrow M^{\prime}$ satisfying $N=\operatorname{ker}(\alpha)$.

Proof: Suppose there exists an $(R, S)$ - homomorphism $\alpha: M \rightarrow M^{\prime}$ such that $N=\operatorname{ker}(\alpha)$. To prove that $\operatorname{ker}(\alpha)$ is a subbi-semimodule of $M$, let $\left(x_{i}: i \in I\right)$ be a summable family in $M$ э each $x_{i} \in \operatorname{ker}(\alpha) . \Rightarrow \alpha\left(x_{i}\right)=0$. $\Rightarrow \bar{\Sigma} \alpha\left(x_{i}\right)=0 . \Rightarrow \alpha\left(\bar{\Sigma}_{i} x_{i}\right)=0 . \Rightarrow \bar{\Sigma}_{i} x_{i} \in \operatorname{ker}(\alpha) \quad$. Let $\quad r \in R \quad, \quad s \in S \quad$ and $\quad x \in \operatorname{ker}(\alpha)$. Then $\alpha(r * x * s)=r * \alpha(x) * s=r * 0_{M} * s=0_{M} . \Rightarrow r * x * s \in \operatorname{ker}(\alpha)$. Hence $\operatorname{ker}(\alpha)$ is a subbi-semimodule of $M$. To prove that $N$ is subtractive, let $x, y \in M \ni x+y \in N$ and $x \in N$. Then $\alpha(x+y)=0$ and $\alpha(x)=0$. $\Rightarrow \alpha(y)=0 . \Rightarrow y \in \operatorname{ker}(\alpha)=N$. Hence $N$ is a subtractive subbi-semimodule of $M$.

Conversely suppose that $N$ is a subtractive subbi-semimodule of $M$. Let $M^{\prime}=M / N$ and define $\alpha: M \rightarrow M^{\prime}$ by 
$m \mapsto m / N$. Now we prove that $\alpha$ is an $(R, S)$ - homomorphism.

Let $\left(m_{i}: i \in I\right)$ be a summable family in $M$. Then $\alpha\left(\sum_{i} m_{i}\right)=\bar{\Sigma}_{i} \alpha m_{i}=\left(\bar{\Sigma}_{i} m_{i}\right) / N$. Therefore $\alpha$ is an additive mapping. Let $r \in R, s \in S$ and $m \in M$. Then $\alpha(r * m * s)=(r * m * s) / N=r * m / N * s=r * \alpha(m) * s$. Hence $\alpha$ is an $(R, S)$ - homomorphism. Now $\operatorname{ker}(\alpha)=\{m \in M \mid \alpha(m)=0 / N\}=\{m \in M \mid m / N=0 / N\}$ $=\{m \in M / \exists x, y \in N$ э $m+x=0+y\}=$

$\{m \in M \mid m \in N\}=N$. Hence the theorem.

4.15. Theorem. Let $\alpha: M \rightarrow N$ be an $(R, S)$ - homomorphism of complete $(R, S)$ - partial bi-semimodules. If $N^{\prime}$ is a subtractive subbi-semimodule of $N$ and $M^{\prime}=\alpha^{-1} N^{\prime} \subseteq M$, then

i. $\quad M^{\prime}$ is a subtractive subbi-semimodule of $M$ containing $\operatorname{ker}(\alpha)$

ii. $\quad \alpha$ induces an $(R, S)$ - homomorphism $\beta: M / M^{\prime} \rightarrow N / N^{\prime}$ having $\operatorname{ker}(\beta)=\left\{0 / M^{\prime}\right\}$.

Proof: (i) Note that $M^{\prime}=\alpha^{-1} N^{\prime}=\left\{m \in M \mid \alpha m \in N^{\prime}\right\}$ is a subbi-semimodule of $M$. To prove that $M^{\prime}$ is subtractive, let $m, m^{\prime} \in M \ni m+m^{\prime} \in M^{\prime}$ and $m \in M^{\prime} . \Rightarrow \alpha m+\alpha m^{\prime} \in N^{\prime}$ and $\alpha m \in N^{\prime}$. Since $N^{\prime}$ is subtractive, $\alpha m^{\prime} \in N^{\prime} . \Rightarrow m^{\prime} \in \alpha^{-1} N^{\prime}=M^{\prime}$. Therefore $M^{\prime}$ is subtractive. To prove that $\operatorname{ker}(\alpha) \subseteq M^{\prime}$, let $m \in \operatorname{ker}(\alpha)$. Then $\alpha m=0 \in N^{\prime} . \Rightarrow m \in \alpha^{-1} N^{\prime}=M^{\prime} . \Rightarrow \operatorname{ker}(\alpha) \subseteq M^{\prime}$.

(ii) Define $\beta: M / M^{\prime} \rightarrow N / N^{\prime}$ by $m / M^{\prime} \mapsto \alpha m / N^{\prime}$. First we prove that $\beta$ is well defined. Let $x / M^{\prime}, y / M^{\prime} \in M / M^{\prime} \ni x / M^{\prime}=y / M^{\prime} \quad$. Then $\quad m, m^{\prime} \in M^{\prime} \ni x+m=y+m^{\prime}$

$\Rightarrow \exists \alpha m, \alpha m^{\prime} \in N^{\prime} \ni \alpha x+\alpha m=\alpha y+\alpha m^{\prime} . \Rightarrow \alpha x / N^{\prime}=\alpha y / N^{\prime}$. Hence $\beta$ is well defined. Now we prove that $\beta$ is an $(R, S)$ - homomorphism. Let $\left(x_{i} / M^{\prime}: i \in I\right)$ be a summable family in $M / M^{\prime}$. Then $\left(\alpha x_{i} / N^{\prime}: i \in I\right)$ is a summable family in $N / N^{\prime} . \Rightarrow\left(\beta\left(x_{i} / M^{\prime}\right): i \in I\right)$ is a summable family in $N / N^{\prime}$. Consider $\beta\left(\bar{\Sigma}_{i}^{\prime}\left(x_{i} / M^{\prime}\right)\right)=\beta\left(\left(\bar{\Sigma} x_{i}\right) / M^{\prime}\right)=\alpha\left(\bar{\Sigma}_{i} x_{i}\right) / N^{\prime}=\bar{\Sigma}_{i}^{\prime}\left(\alpha x_{i} / N^{\prime}\right)=\bar{\Sigma}_{i}^{\prime} \beta\left(x_{i} / M^{\prime}\right)$. Let $r \in R, s \in S \quad$ and $x / M^{\prime} \in M / M^{\prime} \quad$. Consider $\quad \beta\left(\left(r *\left(x / M^{\prime}\right) * s\right)=\beta\left((r * x * s) / M^{\prime}\right)=\alpha(r * x * s) / N^{\prime}=\right.$ $r * \alpha(x) / N^{\prime} * s=r * \beta\left(x / M^{\prime}\right) * s$. Hence $\beta$ is an $(R, S)-$ homomorphism. To prove $\operatorname{ker}(\beta)=\left\{0 / M^{\prime}\right\}$, let $x / M^{\prime} \in \operatorname{ker}(\beta)$. Then $\beta\left(x / M^{\prime}\right)=0 / N^{\prime} . \Rightarrow \alpha x / N^{\prime}=0 / N^{\prime} . \Rightarrow \exists a, b \in N^{\prime} \ni \alpha x+a=b \in N^{\prime}$. Since $N^{\prime}$ is subtractive, $\alpha x \in N^{\prime} . \Rightarrow x \in \alpha^{-1} N^{\prime}=M^{\prime} . \Rightarrow x / M^{\prime}=0 / M^{\prime} . \Rightarrow \operatorname{ker}(\beta)=\left\{0 / M^{\prime}\right\}$. Hence the theorem.

4.16. Definition. Let $\alpha: M \rightarrow M^{\prime}$ be an $(R, S)$-homomorphism of $(R, S)$ - partial bi-semimodules then a relation $\equiv_{\alpha}$ on $M$ is defined as $m \equiv_{\alpha} m^{\prime} \Leftrightarrow \alpha(m)=\alpha\left(m^{\prime}\right)$.

4.17. Remark. The relation $\equiv_{\alpha}$ is an $(R, S)$ - congruence relation on an $(R, S)$ - partial bi-semimodule $M$.

Proof: Let $\left(x_{i}: i \in I\right)$ and $\left(y_{i}: i \in I\right)$ be summable families in $M$ such that $\left(x_{i}, y_{i}\right) \in \equiv_{\alpha} . \Rightarrow_{x_{i}} \equiv_{\alpha} y_{i}$. $\Rightarrow \alpha\left(x_{i}\right)=\alpha\left(y_{i}\right) \forall i \in I . \Rightarrow \bar{\Sigma} \alpha\left(x_{i}\right)=\bar{\Sigma} \alpha\left(y_{i}\right) . \Rightarrow \alpha\left(\bar{\Sigma} x_{i}\right)=\alpha\left(\bar{\Sigma} y_{i}\right) . \Rightarrow \sum_{i} x_{i} \equiv_{\alpha} \bar{\Sigma} y_{i} . \Rightarrow\left(\bar{\Sigma} x_{i}, \bar{\Sigma} y_{i}\right) \in \equiv_{\alpha}$. $\Rightarrow \equiv_{\alpha}$ is closed under additive operation.

Let $\quad r \in R \quad$ and $s \in S, \quad(x, y) \in \equiv_{\alpha} . \Rightarrow x \equiv_{\alpha} y . \Rightarrow \alpha(x)=\alpha(y)$. Consider $\alpha(r * x * s)=$ $r * \alpha(x) * s=r * \alpha(y) * s=\alpha(r * y * s) . \Rightarrow r * x * s \equiv_{\alpha} r * y * s . \Rightarrow(r * x * s, r * y * s) \in \equiv_{\alpha}$.

Hence $\equiv_{\alpha}$ is an $(R, S)$ - congruence relation on $M$.

If $M$ is a complete $(R, S)$ - partial bi-semimodule and $\alpha: M \rightarrow M^{\prime}$ is an $(R, S)$ - homomorphism, then clearly $\equiv_{\operatorname{ker}(\alpha)} \subseteq \equiv_{\alpha}$. The following example shows that $\equiv_{\alpha}$ need not be contained in $\equiv_{\operatorname{ker}(\alpha)}$.

4.18. Example. Let $R$ be the so-ring $\{0,1\}$ with trivial addition and trivial multiplication. Any partial monoid $(M, \bar{\Sigma})$ is uniquely an $(R, R)$ - partial bi-semimodule with ' $*$ ' defined for any $x \in M$ as $1 * x * 1=x$, $0 * x * 0=0 * x * 1=1 * x * 0=0$. Consider the partial monoid $M_{1}=\{0, a, b, 1\}$ with $\Sigma$ on $M_{1}$ defined by 
$\bar{\Sigma} x_{i}=\left\{\begin{array}{ccc}x_{j}, & \text { if } x_{i}=0 \quad \forall i \neq j \quad \text { for some } j \\ \text { undefined, } & \text { otherwise }\end{array}\right.$. Then $\left(M_{1}, \bar{\Sigma}\right)$ is an $(R, R)$ - partial bi-semimodule and the mapping $h: M_{1} \rightarrow M_{2}$ defined by $0 \mapsto 0, a \mapsto 1, b \mapsto 1$ and $1 \mapsto 1$ is an $(R, S)$ - homomorphism. Now $(a, b) \in \equiv_{h}$ but $(a, b) \notin \equiv_{\operatorname{ker}(h)}$.

4.19. Definition. Let $\alpha: M \rightarrow M^{\prime}$ be an $(R, S)$ - homomorphism of complete $(R, S)$ - partial bi-semimodules. Then $\alpha$ is said to be a steady $(R, S)$ - homomorphism if the relations $\equiv_{\alpha}$ and $\equiv_{\operatorname{ker}(\alpha)}$ coincide.

4.20. Remark. A steady $(R, S)$ - homomorphism $\alpha$ is monic if and only if $\operatorname{ker}(\alpha)=\{0\}$.

Proof: Suppose $\alpha: M \rightarrow N$ is a steady $(R, S)$ - homomorphism and monic. Then for any $m \in \operatorname{ker}(\alpha), \alpha(m)=0_{N}=\alpha\left(0_{M}\right) \quad . \Rightarrow m=0_{M} \quad$ and hence $\operatorname{ker}(\alpha)=\{0\}$. Conversely suppose that $\operatorname{ker}(\alpha)=\{0\} \quad$ and let $m, m^{\prime} \in M$ э $\alpha(m)=\alpha\left(m^{\prime}\right)$. Then $m \equiv_{\alpha} m^{\prime} . \Rightarrow m \equiv_{\operatorname{ker}(\alpha)} m^{\prime} . \Rightarrow m \equiv_{\{0\}} m^{\prime}$. $\Rightarrow m=m^{\prime}$. Hence $\alpha$ is monic.

4.21. Theorem. If $\alpha$ is a steady $(R, S)$ - endomorphism of a complete $(R, S)$-partial bi-semimodule $M$ then $\alpha^{K}$ is steady for each $k \geq 1$.

Proof: Suppose that $\alpha^{K}$ is steady and we prove $\alpha^{K+1}$ is steady. Note that $\alpha^{K+1}$ is an $(R, S)$-endomorphism on $M$. Let $m \equiv_{\alpha^{k+1}} m^{\prime}$. Then $\quad \alpha^{k+1}(m)=\alpha^{k+1}\left(m^{\prime}\right) \quad . \Rightarrow \alpha\left(\alpha^{k}(m)\right)=\alpha\left(\alpha^{k}\left(m^{\prime}\right)\right) \quad . \quad \Rightarrow \alpha^{K}(m) \equiv_{\alpha} \alpha^{k}\left(m^{\prime}\right)$. $\Rightarrow \alpha^{k}(m) \equiv_{\operatorname{ker}(\alpha)} \alpha^{k}\left(m^{\prime}\right) \quad . \quad \Rightarrow(m+x) \equiv_{\operatorname{ker}\left(\alpha^{k}\right)}\left(m^{\prime}+x^{\prime}\right) \quad$ for $\quad$ some $\quad x, x^{\prime} \in \operatorname{ker}(\alpha)$

$\Rightarrow m+(x+y)=m^{\prime}+\left(x^{\prime}+y^{\prime}\right)$ for some $x+y, x^{\prime}+y^{\prime} \in \operatorname{ker}\left(\alpha^{k+1}\right) . \Rightarrow m \equiv_{\operatorname{ker}\left(\alpha^{k+1}\right)} m^{\prime}$. Hence $\alpha^{K+1}$ is steady. Hence the statement is true by the induction.

4.22. Theorem. Let $N^{\prime} \subseteq N$ be subbi-semimodules of a complete $(R, S)$-partial bi-semimodule $M$. Then the function $\alpha: M / N^{\prime} \rightarrow M / N$ defined by $m / N^{\prime} \mapsto m / N$ is a steady surjective $(R, S)$ - homomorphism.

Proof: The mapping $\alpha: M / N^{\prime} \rightarrow M / N$ defined by $m / N^{\prime} \mapsto m / N$ is clearly a surjective $(R, S)$ - homomorphism. To prove $\alpha$ is steady, let $m / N^{\prime} \equiv_{\alpha} m^{\prime} / N^{\prime}$. Then $\alpha\left(m / N^{\prime}\right)=\alpha\left(m^{\prime} / N^{\prime}\right) . \Rightarrow m / N=m^{\prime} / N$. $\Rightarrow \exists x, y \in N \ni m+x=m^{\prime}+y . \Rightarrow(m+x) / N^{\prime}=\left(m^{\prime}+y\right) / N^{\prime}$.

$\Rightarrow \exists x / N^{\prime}, y / N^{\prime} \in \operatorname{ker}(\alpha)$ э $m / N^{\prime}+x / N^{\prime}=m^{\prime} / N^{\prime}+y / N^{\prime} . \Rightarrow m / N^{\prime} \equiv_{\operatorname{ker}(\alpha)} m^{\prime} / N^{\prime}$. Hence $\alpha$ is a steady $(R, S)$ - homomorphism.

4.23. Definition. A surjective $(R, S)$ - homomorphism $\alpha: M \rightarrow N$ is said to be an $(R, S)$-semiisomorphism if and only if $\operatorname{ker}(\alpha)=\left\{0_{M}\right\}$.

4.24. Example. Consider the $(R, R)$ - partial bi-semimodules $M_{1}=\{0, a, b, 1\}$ and $M_{2}=R=\{0,1\}$ as in the example 4.18. Then the mapping $\alpha: M_{1} \rightarrow M_{2}$ defined by $\alpha: 0 \mapsto 0, a \mapsto 1, b \mapsto 1,1 \mapsto 1$ is a surjective $(R, S)$ homomorphism such that $\operatorname{ker}(\alpha)=\{0\}$ and hence $\alpha$ is an $(R, S)$-semiisomorphism.

4.25. Corollary. Let $\alpha: M \rightarrow N$ be a surjective $(R, S)$-homomorphism of a complete $(R, S)$-partial bi-semimodule. Then there exists an $(R, S)$-semiisomorphism $M / \operatorname{ker}(\alpha) \rightarrow N$.

Proof: Note that $N^{\prime}=\{0\}$ is a subtractive subbi-semimodule of $N$ and $M^{\prime}=\alpha^{-1} N^{\prime}=\left\{m \in M / \alpha m \in N^{\prime}\right\}=\operatorname{ker}(\alpha)$. By the theorem 4.15, $\exists$ an $(R, S)$ - homomorphism $\beta: M / M^{\prime} \rightarrow N / N^{\prime}$ having $\operatorname{ker}(\beta)=\{0 / M\} . \Rightarrow \exists$ an $(R, S)$ - homomorphism $\beta: M / \operatorname{ker}(\alpha) \rightarrow N \ni \operatorname{ker}(\beta)=\{0 / \operatorname{ker}(\alpha)\} \quad$ and $\quad \beta \quad$ is surjective. $\Rightarrow \exists$ an $(R, S)$ -semiisomorphism $\beta: M / \operatorname{ker}(\alpha) \rightarrow N$.

4.26. Corollary. If $N^{\prime} \subseteq N$ be subbi-semimodules of a complete $(R, S)$-partial bi-semimodule $M$ then $M / N$ is $(R, S)$ - semiisomorphic to $\left(M / N^{\prime}\right) /\left(N / N^{\prime}\right)$.

Proof: The mapping $\alpha: M / N^{\prime} \rightarrow M / N$ defined by $m / N^{\prime} \mapsto m / N$ is clearly surjective $(R, S)$ homomorphism with $\operatorname{ker}(\alpha)=N / N^{\prime}$. Hence by above corollary, $\left(M / N^{\prime}\right) /\left(N / N^{\prime}\right)$ is $(R, S)$ - semiisomorphic to $M / N$. 
4.27. Corollary. If $N$ and $N^{\prime}$ are subbi-semimodules of a complete $(R, S)$ - partial bi-semimodule $M$ then there exists a canonical surjective $(R, S)$ - homomorphism $\alpha: N^{\prime} /\left(N \cap N^{\prime}\right) \rightarrow\left(N+N^{\prime}\right) / N \quad$ and $\alpha$ is an $(R, S)$ semiisomorphism if $N$ is subtractive.

Proof: The mapping $\alpha: N^{\prime} /\left(N \cap N^{\prime}\right) \rightarrow\left(N+N^{\prime}\right) / N$ defined by $n^{\prime} /\left(N \cap N^{\prime}\right) \mapsto n^{\prime} / N$ is a surjective $(R, S)$ homomorphism. Suppose $N$ is subtractive and let $n^{\prime} / N \cap N^{\prime} \in \operatorname{ker}(\alpha) \quad \Rightarrow n^{\prime} / N=0 / N$. $\Rightarrow \exists n_{1}, n_{2} \in N \ni n^{\prime}+n_{1}=0+n_{2} \in N . \Rightarrow n^{\prime} \in N$. Hence $\operatorname{ker}(\alpha)=\left\{0 / N \cap N^{\prime}\right\}$. Then by above corollary, $\alpha$ is an $(R, S)$-semiisomorphism.

4.28. Definition. A non zero subbi-semimodule $N$ of an $(R, S)$ - partial bi-semimodule $M$ is said to be absorbing ( denoted as $N \prec M$ ) if and only if ( i ) if $0_{M} \neq n \in N$ and $m \in M$ then $0_{M} \neq n+m \in N$, and ( ii ) if $0_{M} \neq n \in N$ then $\left(0_{M}: n\right)_{R}=\left\{0_{R}\right\}$.

4.29. Theorem. Let $M$ be an $(R, S)$ - partial bi-semimodule and let $\left\{N_{j} \mid j \in J\right\}$ be a family of absorbing subbi-semimodules of $M$ such that $\bigcap_{j \in J} N_{j}=N$ and $\bigcup_{j \in J} N_{j}=N^{\prime}$. Then $N \prec M$ and $N^{\prime} \prec M$.

Proof: Note that $N=\bigcap_{j \in J} N_{j}$ is a subbi-semimodule of $M$. Now let $0 \neq n \in N$ and $m \in M$. Then $0 \neq n \in N_{j}$ $\forall j \in J$ and $m \in M . \Rightarrow 0 \neq n+m \in N_{j} \quad \forall j \in J$ and hence $0 \neq n+m \in N$. Let $0 \neq n \in N$. Then $0 \neq n \in N_{j} \quad \forall j \in J$ and hence $\left(0_{M}: n\right)_{R}=\left\{0_{R}\right\}$. Hence $N \prec M$. Now we prove that $N^{\prime}=\bigcup_{j \in J} N_{j}$ is a subbi-semimodule of $M$. Let $\left(x_{i}: i \in I\right)$ be a summable family in $M$ and $x_{i} \in N^{\prime} \quad \forall i \in I$. Then for each $i \in I, x_{i} \in N_{j_{i}} \quad$ for $\quad$ some $\quad j_{i} \in J \quad$ and $\quad \bar{\Sigma}_{i} x_{i}$ exists in $\quad M$. Now $0 \neq x_{i} \in N_{j_{i}}$ and $\overline{\sum_{k \neq i}} x_{k} \in M$. $\Rightarrow 0 \neq x_{i}+\overline{\sum_{k \neq i}} x_{k} \in N_{j_{i}}$ for some $j_{i} \in J \quad\left(\because N_{j_{i}} \prec M\right) . \Rightarrow \bar{\Sigma} x_{i} \in \bigcup_{j \in J} N_{j}$ and hence $\bar{\Sigma} x_{i} \in N^{\prime}$. Now let $r \in R, s \in S$ and $n \in N^{\prime}$. Then $r \in R, s \in S$ and $n \in N_{j}$ for some $j \in J . \Rightarrow r * n * s \in N_{j}$ for some $j \in J$. $\Rightarrow r * n * s \in N^{\prime}$. Hence $N^{\prime}$ is a subbi-semimodule of $M$. Now we prove that $N^{\prime} \prec M$. Let $0 \neq n \in N^{\prime}$ and $m \in M$. Then $0 \neq n \in N_{j}$ for some $j \in J$ and $m \in M . \Rightarrow 0 \neq n+m \in N_{j}$ for some $j \in J$ and hence $0 \neq n+m \in N^{\prime}$. Let $0 \neq n \in N^{\prime}$. Then $0 \neq n \in N_{j}$ for some $j \in J$ and hence $\left(0_{M}: n\right)_{R}=\left\{0_{R}\right\}$. Hence $N^{\prime} \prec M$.

4.30. Remark. If $N$ and $N^{\prime}$ are subbi-semimodules of an $(R, S)$ - partial bi-semimodule $M$. Then we have the following:

(i) if $N \prec M$ and $N \subseteq N^{\prime}$ then $N \prec N^{\prime}$,

(ii ) if $N, N^{\prime} \prec M$ then $N \cap N^{\prime} \neq 0_{M}, N+N^{\prime} \prec M$ and $N+N^{\prime}=N \cup N^{\prime}$, and ( iii ) if $N \prec M$ then $N \cap N^{\prime} \prec N^{\prime}$.

Proof: (i) Suppose $N \prec M$ and $N \subseteq N^{\prime}$. Since $N$ and $N^{\prime}$ are subbi-semimodules of $M$ and $N \subseteq N^{\prime}$, we have $N$ is a subbi-semimodule of $N^{\prime}$. Let $0 \neq n \in N$ and $m \in N^{\prime}$. Then $0 \neq n \in N$ and $m \in M$ and hence $0 \neq n+m \in N$. Let $0 \neq n \in N$. Then $\left(0_{M}: n\right)_{R}=\left\{0_{R}\right\}$. Hence $N \prec N^{\prime}$.

( ii ) Suppose $N \prec M$ and $N^{\prime} \prec M$. Then $\exists 0 \neq n \in N$ and $0 \neq n^{\prime} \in N^{\prime} \ni 0 \neq n+n^{\prime} \in N$ and $0 \neq n^{\prime}+n \in N^{\prime}$. Hence $N \cap N^{\prime} \neq 0_{M}$. Now we prove that $N+N^{\prime} \prec M$. Note that $N+N^{\prime}$ is a subbi-semimodule of $M$. Let $0 \neq x \in N+N^{\prime}$ and $m \in M . \Rightarrow 0 \neq x=n+n^{\prime}$ for some $n \in N, n^{\prime} \in N^{\prime}$ and $m \in M$. Since $N \prec M$, we have $0 \neq n+m \in N$. Since $N^{\prime} \prec M, 0 \neq n^{\prime} \in N^{\prime}$ and $0 \neq n+m \in M$, we have $0 \neq n^{\prime}+n+m \in N^{\prime} \quad . \quad \Rightarrow 0 \neq\left(n+n^{\prime}\right)+m \in N^{\prime} \subseteq N+N^{\prime} \quad$ and hence $0 \neq x+m \in N+N^{\prime}$. Let $0 \neq x \in N+N^{\prime}$. Then $0 \neq x=n+n^{\prime} \quad$ for $\quad$ some $n \in N, n^{\prime} \in N^{\prime} . \Rightarrow\left(0_{M}: n\right)_{R}=\left\{0_{R}\right\} \quad$ and $\left(0_{M}: n^{\prime}\right)_{R}=\left\{0_{R}\right\}$ and hence $\left(0_{M}: x\right)_{R}=\left\{0_{R}\right\}$. Hence $N+N^{\prime} \prec M$. Clearly $N \cup N^{\prime} \subseteq N+N^{\prime}$. Let $0 \neq x \in N+N^{\prime}$. Then $0 \neq x=n+n^{\prime}$ for some $n \in N, n^{\prime} \in N^{\prime} . \Rightarrow 0 \neq n \in N$ and $0 \neq n^{\prime} \in N^{\prime}$. 
$\Rightarrow 0 \neq n+n^{\prime} \in N$ and $0 \neq n^{\prime}+n \in N^{\prime}$ and hence $x \in N \cup N^{\prime}$. Hence $N+N^{\prime}=N \cup N^{\prime}$.

( iii ) Suppose $N \prec M$ and let $0 \neq x \in N \cap N^{\prime}$ and $n^{\prime} \in N^{\prime}$. Then $0 \neq x \in N, x, n^{\prime} \in N^{\prime}$. $\Rightarrow 0 \neq x+n^{\prime} \in N$ and $x+n^{\prime} \in N^{\prime}$. Hence $0 \neq x+n^{\prime} \in N \cap N^{\prime}$. Now let $0 \neq n \in N \cap N^{\prime}$. Then $0 \neq n \in N$ and hence $\left(0_{M}: n\right)_{R}=\left\{0_{R}\right\}$. Hence $N \cap N^{\prime} \prec N^{\prime}$.

4.31. Theorem. Let $\alpha: M \rightarrow M^{\prime}$ be an $(R, S)$ - homomorphism of ( $R, S$ )- partial bi-semimodules satisfying the condition $\operatorname{ker}(\alpha) \prec M$. If $N^{\prime} \prec M^{\prime}$ then $N=\alpha^{-1} N^{\prime} \prec M$.

Proof: Suppose $N^{\prime} \prec M^{\prime}$. Note that $N=\alpha^{-1} N^{\prime}=\left\{x \in M \mid \alpha(x) \in N^{\prime}\right\}$ is a subbi-semimodule of $M$. Now we prove that $N \prec M$. Let $n \in N \ni n \neq 0$ and $m \in M$. Suppose $n \in \operatorname{ker}(\alpha)$. Since $n \in \operatorname{ker}(\alpha), m \in M$ and $\operatorname{ker}(\alpha) \prec M$, we have $0 \neq n+m \in \operatorname{ker}(\alpha) . \Rightarrow \alpha(n+m)=0 \in N^{\prime} . \Rightarrow 0 \neq n+m \in N$. Suppose $n \notin \operatorname{ker}(\alpha)$. Then $\alpha(n) \neq 0$. Since $N^{\prime} \prec M^{\prime}, \quad 0 \neq \alpha(n) \in N^{\prime}$ and $\alpha(m) \in M^{\prime}$, we have $0 \neq \alpha(n)+\alpha(m) \in N^{\prime}$. $\Rightarrow 0 \neq \alpha(n+m) \in N^{\prime}$. Hence $0 \neq n+m \in N$. Let $n \in N \ni n \neq 0$. Suppose $n \in \operatorname{ker}(\alpha)$. Since $\operatorname{ker}(\alpha) \prec M$, we have $\left(0_{M}: n\right)_{R}=\left\{0_{R}\right\}$. Suppose $n \notin \operatorname{ker}(\alpha) . \Rightarrow 0 \neq \alpha n \in N^{\prime}$. Let $r \in\left(0_{M}: n\right)_{R}$. Then $r * n * S=0$. $\Rightarrow r * n * s=0 \forall s \in S \quad . \quad \Rightarrow \alpha(r * n * s)=0 \quad . \quad \Rightarrow r * \alpha(n) * s=0 \forall s \in S \quad . \quad \Rightarrow r * \alpha(n) * S=0$. $\Rightarrow r \in\left(0_{M}: \alpha(n)\right)$. Since $N^{\prime} \prec M^{\prime}$, we have $\left(0_{M}: \alpha(n)\right)_{R}=\left\{0_{R}\right\} . \Rightarrow r=0$. Hence $\left(0_{M}: n\right)_{R}=\left\{0_{R}\right\}$. Hence $N=\alpha^{-1} N^{\prime} \prec M$.

\section{Conclusion}

In view of partial addition in the partial semirings, the bi-semimodule theory of semirings( Jonathan S. Golan[4]) are not applicable to partial semirings and hence we introduced the notions of $(R, S)$ - partial bi-semimodule, $(R, S)$-homomorphism of $(R, S)$ - partial bi-semimodules, $(R, S)$-semiisomorphism and absorbing subbi-semimodule and generalised the results of semirings( Jonathan S. Golan[4]) and results of partial semirings( Srinivasa Rao. P. V [9]) to the class of $(R, S)$ - partial bi-semimodules of partial semirings. In this investigation we studied the characteristics of the set $(N: M)$, proved that $(0$ : $M)_{R}=(0: m)_{R}$ for every nonzero $m$ in $M$ where $M$ is a bi-austere $(R, S)$ - partial bi-semimodule and obtained the characterization of subtractive subbi-semimodules by an $(R, S)$ - homomorphism. Also we obtained the semiisomorphism theorems for $(R, S)$ - partial bi-semimodules and studied the absorbing subbi-semimodules.

\section{REFERENCES}

[1] Acharyulu, G.V.S.: A Study of Sum-Ordered Partial Semirings, Doctoral thesis, Andhra University,1992.

[2] Amarendra Babu, V., and Srinivasa Reddy, M.: Bi-ideals of Sum-Ordered Partial Semirings, International Journal of Mathematics and Computer Applications Research(IJMCAR), Vol.3, issue 2, pp. 57-64, June 2013.

[3] Arbib, M.A.and Manes, E.G.: Partially Additive Categories and Flow-diagram Semantics, Journal of Algebra, vol. 62, pp. 203-227, 1980 .

[4] Jonathan S. Golan: Semirings and their Applications, Kluwer Academic Publishers, 1999.

[5] Le Roux, H.J.: A Note on Prime and Semiprime Bi-ideals, Kyungpook Math. J., Vol. 35, pp. 243-247, 1995.

[6] Manes, E.G., and Benson, D.B.: The Inverse Semigroup of a Sum-Ordered Semiring, Semigroup Forum, vol. 31, pp. 129-152, 1985.

[7] Streenstrup, M.E.: Sum-Ordered Partial Semirings, Doctoral thesis, Graduate school of the University of Massachusetts, Feb 1985 (Department of computer and Information Science).

[8] Srininvasa Rao, P.V.: Partial semimodules over Partial Semirings, Intenational Journal of Computational Cognition(IJCC), Vol. 8, No. 4, pp. 80-84, Dec 2010.

[9] Srininvasa Rao, P.V.: Ideal Theory Of Sum-Ordered Partial Semirings, Doctoral thesis, Acharya Nagarjuna University, 2011.

[10] Srinivasa Reddy, M., Amarendra Babu, V., and Srinivasa Rao, P. V.: Prime and Semiprime bi- ideals of So- rings, International Journal of Scientific and Innovative Mathematical Research (IJSIMR), vol. 1, issue 2, pp.134-143, oct 2013. 